\title{
MODELING AND IDENTIFICATION OF ROBOT DYNAMiC PARAMETERS. RESOLUTIONS OF THE InVERSE DyNAMic Problem in Real-Time
}

\author{
Rosillo, N.; VAlera, A.; Benimeli, F.; MATA, V. \& VAlero, F.
}

Abstract: This work presents the development and validation of a methodology allowing dynamic parameter identification in robotic manipulators. The dynamic equations of the robot are based on the Gibbs-Appell equations of motion, yielding well structured equations which can be computed in real time and make the model easily expandable, which allows to include the actuator dynamic effect and the friction phenomenon.

This paper also deals with dynamic modeling identification in robotic manipulators. Two different methods are proposed and validated on an industrial robotic system: a PUMA 560 provided with an open control architecture based on an industrial personal computer. Finally, using the dynamic parameters identified, the inverse dynamic equations have been obtained and implemented in real time.

Key words: Dynamic parameter identification; Gibbs-Appell equations; Robot Control; Robot Modeling, Friction model
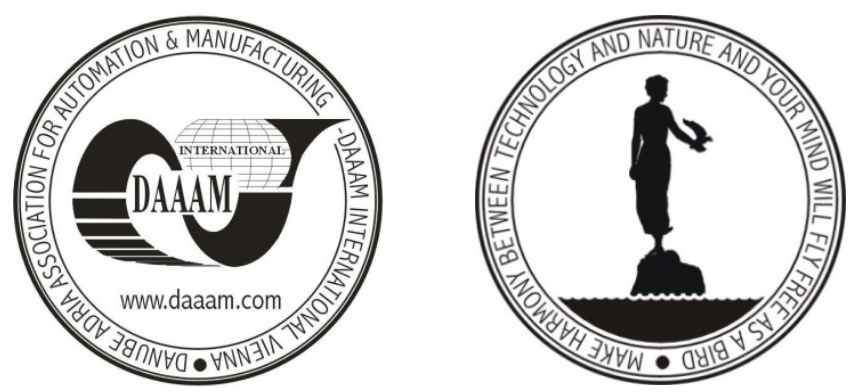

Authors' data: Prof. Rosillo, N[uria]*; Prof. Valera, A[ngel]**; Dr. Benimeli, F[rancesc]***; Prof. Dr. Mata, V[icente $]^{* * * *}$; Dr. Valero, F[rancisco] ${ }^{* * * * *}$, *Universidad Cardenal HerreraCEU San Pablo, c/San Bartolomé, 55 (46115 Alfara Patriarca), 46115, Valencia, ES, ** Universidad Politecnica de Valencia, Dpto. Ingeniería de Sistemas y Automática, 46022, Valencia, ES, *** Universitat Politècnica de València, Institut d'Automàtica i Informàtica Industrial, 46022, València, ES, ****Departamento de Ingeniería Mecánica y de Materiales. Universidad Politécnica de Valencia, C/ Troya 4, 10², 46007, Valencia, ES, *****Universidad Politecnica de Valencia, Camino de Vera s/n, 46022, Valencia, ES, frabean@ai2.upv.es, fvalero@mcm.upv.es, giuprog@isa.upv.es, nrosillo@uch.ceu.es, vmata@mcm.upv.es

This Publication has to be referred as: Rosillo, N[uria]; Valera, A[ngel]; Benimeli, F[rancesc]; Mata, V[icente] \& Valero, F[rancisco] (2008). Modeling and Identification of Robot Dynamic Parameters. Resolutions of the Inverse Dynamic Problem in Real-Time, Chapter 56 in DAAAM International Scientific Book 2008, pp. 657-672, B. Katalinic (Ed.), Published by DAAAM International, ISBN 978-3-901509-66-7, ISSN 1726-9687, Vienna, Austria

DOI: $10.2507 /$ daaam.scibook.2008.56 